\title{
Mid-infrared imaging using upconversion - Principles and applications
}

\author{
Tidemand-Lichtenberg, Peter; Rodrigo, Peter John; Pedersen, Christian
}

Published in:

Mid-Infrared Coherent Sources 2018

Link to article, DOI:

10.1364/MICS.2018.MM4C.1

Publication date:

2018

Document Version

Peer reviewed version

Link back to DTU Orbit

Citation (APA):

Tidemand-Lichtenberg, P., Rodrigo, P. J., \& Pedersen, C. (2018). Mid-infrared imaging using upconversion Principles and applications. In Mid-Infrared Coherent Sources 2018 (Vol. 2018). [Paper MM4C.1] Optical Society of America (OSA). Optics Infobase Conference Papers https://doi.org/10.1364/MiCS.2018.MM4C.1

\section{General rights}

Copyright and moral rights for the publications made accessible in the public portal are retained by the authors and/or other copyright owners and it is a condition of accessing publications that users recognise and abide by the legal requirements associated with these rights.

- Users may download and print one copy of any publication from the public portal for the purpose of private study or research.

- You may not further distribute the material or use it for any profit-making activity or commercial gain

- You may freely distribute the URL identifying the publication in the public portal 


\title{
Mid-Infrared Imaging using Upconversion - Principles and Applications
}

\author{
Peter Tidemand-Lichtenberg, Peter John Rodrigo and Christian Pedersen \\ DTU Fotonik, Technical University of Denmark, DK-4000 Roskilde, Denmark
}

\begin{abstract}
Different schemes for mid-infrared hyperspectral imaging using upconversion detection is implemented and compared in terms of spectral coverage, field-of-view, resolution and speed. Both broadband and narrowband, continuous wave and pulsed imaging systems are considered. OCIS codes: (110.4234) Multispectral and hyperspectral imaging (110.3080) Infrared imaging (100.3010) Image reconstruction techniques (190.7220) Upconversion (300.6340) Spectroscopy, infrared
\end{abstract}

\section{Introduction}

Infrared spectroscopy and imaging has attracted much attention in the past decade. Particularly the mid-infrared (MIR) spectral region, often referred to as the chemical fingerprint region [1], has been studied intensively as many gasses and other compounds have their fundamental absorption lines in this region. Recent years has shown much improvement in available illumination sources, e.g. Quantum Cascade Lasers (QCL) and SuperContinuum (SC) sources extended into the infrared spectral range. However, a similar development has not been seen for MIR detection systems, where direct detection using low bandgap semiconductor detectors or microbolometers has been the preferred choice, either as scanning point detector or as focal plane arrays [2], often relying on cooling in order to obtain sufficient signal-to-noise ratio.

Considering broadband illumination or emission from an object, the spectral resolution relies on the detection system. MIR hyperspectral imaging is usually accomplished by Fourier transform infrared spectroscopy (FTIR). When the system noise is dominated by the detector, it has been shown that FTIR is the optimal configuration [3].

In this work, we introduce and compare several approaches for hyperspectral MIR imaging all based on frequency upconversion and the use of standard Si-detectors. The frequency upconversion process consists of sumfrequency mixing of the MIR signal with a narrowband laser field, transferring the spatial and spectral information from the MIR to the NIR allowing for direct detection with a Si-camera. The upconversion process relies on energy conservation resulting in the unique transfer of the spectral information. Likewise the momentum conservation insures transfer of spatial information [4].
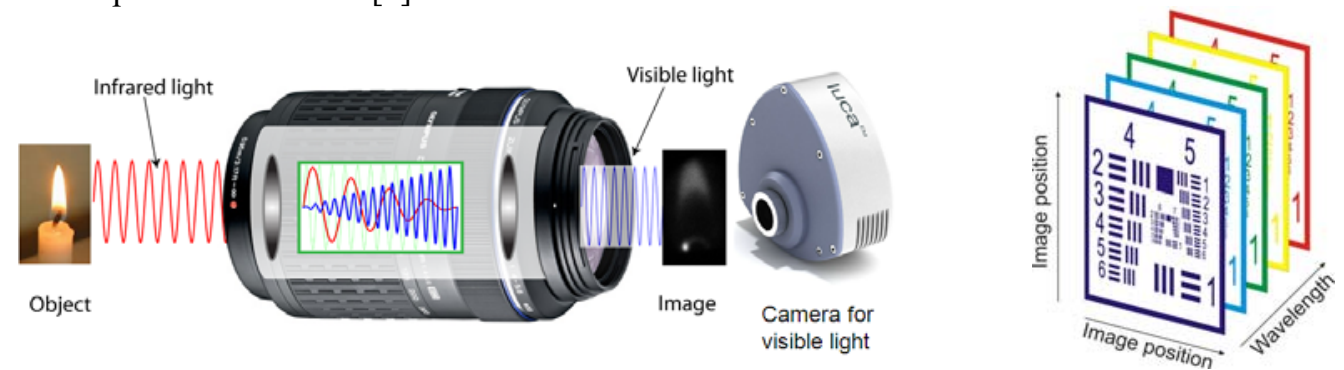

Fig. 1. The principle of upconversion detection, allowing for the use of standard Si-detectors. From the acquired upconverted images post-processing algorithms allow for the extraction of a stack of monochromatic images.

Sum-frequency mixing gives, albeit being a second order nonlinear process, a linear relation between the input MIR signal and the upconverted NIR signal at constant mixing power, with a dynamic range covering single photons to 10's of $\mathrm{mW}$ of MIR signal power. The choice of nonlinear material is very important; the material needs to be transparent at all mixing wavelengths, it should have a high nonlinearity, and be able to phase match the wavelength conversion process. Using a transparent material for the wavelength conversion process means, that blackbody radiation is not generated in the material, hence, not adding noise to the upconverted signal. This enables the use of highly sensitive Si-detectors even at room temperature operation of the nonlinear crystal.

\section{Applications}

Hyperspectral imaging may find applications in a variety of fields ranging from remote sensing (pollution monitoring) [5] to microspectroscopy (digital histopathology) [6]. Hence, the requirements to spatial and spectral resolution may vary significantly depending on the application. 
For remote sensing different schemes can be considered either based on passive detection or active illumination. However, for remote sensing and standoff detection raster scanning is the most efficient approach to achieve image information [7]. We demonstrate spectral imaging of a hot object at a distance of several meters based on raster scanning of the remote target and spectral detection using upconversion allowing for retrieval of the spectral signature of the MIR emission from the target.

We also demonstrate microspectroscopy leading toward histopathology without the need for exogenous staining. This approach relies on chemical specificity from absorption features in tissue biopsies. Here spatial resolution is very important, while the spectral features are relatively broad.

The nature of the MIR signal is very important for the choice of frequency conversion scheme. If the signal is pulsed, the best conversion efficiency and lowest noise is obtained by synchronized pulsed upconversion [8,9], however, if the signal is a continuous wave, then the mixing field should also be continuous wave.

\section{Post-processing}

Using upconversion for spectral imaging, post-processing is needed in order to extract monochromatic images. One either builds the images from raster scanning or from converting the upconverted images to monochromatic images.
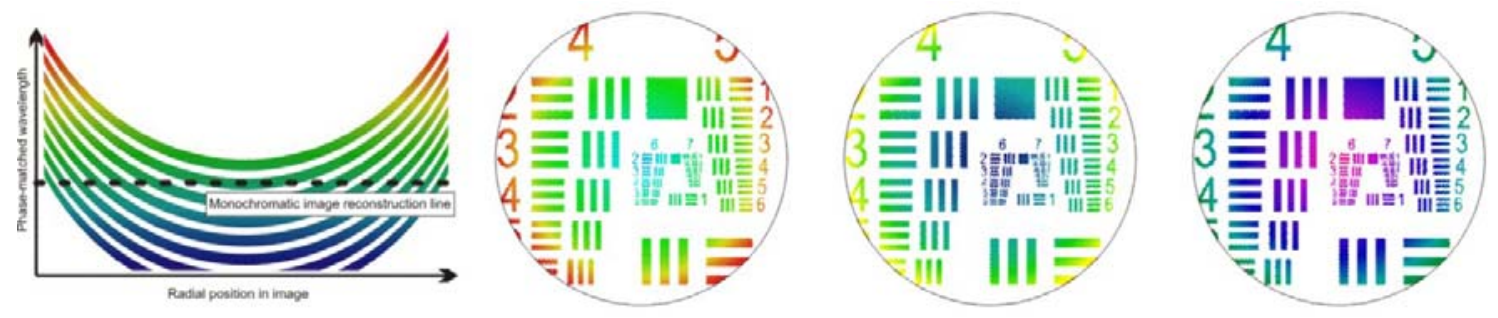

Fig. 2. Scanning of the phase match condition of the frequency conversion process enables acquisition of the spectral response in each pixel element of the image plane. Post-processing is needed to extract monochromatic images [10].

One method relies on scanning of the phase match condition, e.g the temperature of the nonlinear material [10], an example is shown in Fig 2. Alternatively, scanning of the object within the field of view can be used to obtain the full spectral information within each spatially resolvable element of the image.

\section{Discussion}

Different aspects of frequency upconversion for hyperspectral imaging are considered, i.e. spectral coverages, spectral resolution, acquisition speed and noise. Furthermore, different types of MIR signals, continuous wave or pulsed, narrow or broadband, raster scanning or imaging are considered.

\section{Funding}

Mid-TECH H2020-MSCA-ITN-2014 Grant agreement number: 642661.

\section{References}

[1] R. K. Reddy, M. J. Walsh, M. V. Schulmerich, P. S. Carney, and R. Bhargava, "High-Definition Infrared Spectroscopic Imaging," Applied Spectroscopy, 67, 93-105 (2013).

[2] M. C. Phillips and N. Hô, "Infrared hyperspectral imaging using a broadly tunable external cavity quantum cascade laser and microbolometer focal plane array," Opt. Express 16, 1836- 1845 (2008).

[3] P. R. Griffiths and J. A. de Haseth, "Fourier Transform Infrared Spectrometry", 2nd ed. (Wiley, 2007).

[4] J. S. Dam, P. Tidemand-Lichtenberg, C. Pedersen, "Room temperature mid-IR single photon spectral imaging," Nature Photonics, 6, 788-793 (2012).

[5] H. Xia, G. Shentu, M. Shangguan, X. Xia, X. Jia, C. Wang, J. Zhang, J. S. Pelc, M. M. Fejer, Q. Zhang, X. Dou, and J-W Pan, "Long-range micro-pulse aerosol lidar at $1.5 \mu \mathrm{m}$ with an upconversion single-photon detector," Opt. Lett., 40, 1579-1582 (2015).

[6] M. Hermes, R. B. Morrish, L. Huot, L. Meng, S. Junaid, J. Tomko, G. R. Lloyd, W. T. Masselink, P. Tidemand-Lichtenberg, C. Pedersen, F. Palombo, and N. Stone, "Mid-IR hyperspectral imaging for label-free histopathology and cytology," Accepted for Journal of Optics (2017).

[7] D. Stothard, M. Dunn, and C. Rae, "Hyperspectral imaging of gases with a continuous-wave pump-enhanced optical parametric oscillator," Opt. Express 12, 947-955 (2004).

[8] M. Mathez, P.J. Rodrigo, P. Tidemand-Lichtenberg, and C. Pedersen, "Upconversion imaging using short-wave infrared picosecond pulses," Opt. Lett., 42, 579-582 (2017).

[9] L. Huot, P. M. Moselund, P. Tidemand-Lichtenberg, L. Leick, and C. Pedersen, "Upconversion imaging using an all-fiber supercontinuum source," Opt. Lett., 41, 2466-2469 (2016).

[10] L. M. Kehlet, P. Tidemand-Lichtenberg, J. S. Dam, and C. Pedersen, "Infrared upconversion hyperspectral imaging," Opt. Lett. 40, $938-941$ (2015). 